\title{
Der Wandel der Anerkennung von Fehl- und Totgeburt als Geburt eines Kindes
}

\author{
Julia Böcker (iD)
}

Angenommen: 5. Januar 2022

(C) Der/die Autor(en) 2022

Zusammenfassung Das Aufkommen der Alternativbezeichnungen „Kleine Geburt“ und „Stille Geburt“ ist Ausdruck eines Wandels im Umgang mit Fehlgeburt und Totgeburt. Anstoß des Wandels war zivilgesellschaftliches Engagement Betroffener in den 1980er-Jahren, die sich gegen Entrechtungen in den Kliniken und für soziale Sichtbarkeit und Anerkennung des Verlusts einsetzten. Im Beitrag wird argumentiert, dass Fehl- und Totgeburt in Gesellschaft und Geburtshilfe zunehmend als Geburt (und Verlust) eines Kindes behandelt und Betroffene damit als Eltern anerkannt werden. Empirische Grundlage ist ein Datenkorpus aus narrativen Interviews und natürlichen Daten, die sequenzanalytisch und theoriegenerierend ausgewertet wurden. Der mehrdimensionale Wandel wird anhand von Veränderungen im deutschen Recht, in sozialen Medien und in der Geburtshilfe plausibilisiert. Erstens werden Betroffene durch Änderungen des Personenstandsgesetzes für Fehlgeborene symbolisch als Eltern anerkannt. Zweitens entwickelte sich in Online-Foren ein kollektives Selbstverständnis von ,Sternenelternschaft“, das diskursive und institutionelle Veränderungen außerhalb des Internets bewirkte. Daneben legitimieren und normalisieren individuelle Selbstzeugnisse in den sozialen Medien, über Fehl- und Stillgeburt zu sprechen und diese zu zeigen. Drittens werden die professionellen Praktiken der Personalisierung von Stillgeborenen in der Geburtshilfe diskutiert, die nach Totgeburten inzwischen umfänglich, nach Fehlgeburten teilweise, etabliert sind.

Schlüsselwörter Schwangerschaftsverlust - Entrechtete Trauer · Elternschaft · Leibliche Reproduktion · Soziale Bewegungen · Medien 


\title{
The social change towards recognition of pregnancy loss as child birth
}

\begin{abstract}
The coinage of new terms like "Kleine Geburt [little birth]" and "Stille Geburt [silent birth/stillbirth]" is part of an ongoing social change towards recognition of pregnancy loss as child birth. Since the 1980s grassroot initiatives have been engaging for social recognition, for self-determination in clinics and a general right for parents to bury and register their miscarried and stillborn foetuses. In this paper it is argued that miscarriage and stillbirth are increasingly seen and dealt with as birth (and loss) of a person, and thus intended parents are recognized as parents. Qualitative data from narrative interviews and diverse sources such as Blogs and Vlogs has been collected over a period of five years, and a sequential analysis has been applied. The multi-dimensional social change is discussed in three areas: in German legal framework, in social media, and in obstetric care. First, recent changes of the German Civil Status Law for miscarried foetuses contribute to acknowledgement of bereaved parents. Second, online-communities have been establishing a collective identity of "angel parenthood" that brought about discursive and institutional changes outside the internet. Additionally, individual accounts in social media normalize and legitimize to speak about and exhibit experiences of miscarriage and stillbirth. Third, professional personalization of stillborn foetuses in obstetric care is established after perinatal death and stillbirth, but not (yet) after early pregnancy losses.
\end{abstract}

Keywords Stillbirth - Disenfranchised grief · Parenthood - Human reproduction · Social movement $\cdot$ Media

\section{Einleitung}

Am 1. Oktober 2020 teilte das US-amerikanische Model Chrissy Teigen auf Instagram 33,4 Mio. Follower*innen mit, dass ihr drittes Kind Jack in der 20. Schwangerschaftswoche (SSW) tot zur Welt gekommen ist. Der Post wurde über elf Millionen Mal mit ,gefällt mir“ markiert. Die Veröffentlichung der intimen Story mit Schwarzweiß-Fotos der im Krankenhaus kauernden und weinenden Eltern, die auf einem der Bilder ein kleines, weißes Bündel im Arm halten (Abb. 1 und 2), ging breit durch die Medien (exemplarisch Knöfel 2020). Auch in der Deutschen Hebammenzeitschrift war das Ereignis zwei Monate später der Aufhänger des Editorials. Darin heißt es, es hätten zwar bereits andere Prominente ihre Fehlgeburten öffentlich gemacht, etwa die Pop-Sängerin Beyoncé 2019 und die englische Herzogin Meghan Markle im November 2020; „,[s]olche Offenbarungen waren etwas Neues. Aber dass nun die ganze Welt so unmittelbar teilnehmen konnte, war ein Schritt weiter.“ (Heimbach 2021).

Die gestiegene Präsenz solcher Erfahrungsgeschichten in den sozialen Medien ist Ausdruck eines Wandels im Umgang mit Schwangerschaften, die nicht in einer „erfolgreichen“ Geburt, sondern mit Fehlgeburt, Totgeburt oder dem Tod des Neugeborenen enden. Weitere Phänomene, die sich im Kontext dieses Wandels beobachten lassen, sind das erfolgreiche Engagement für separate Grabfelder und Denkmale für 
Abb. 1 Selbstzeugnis auf Instagram: Model Chrissy Teigen nach einer Stillgeburt in der 20. SSW, 01.10.2020 (Screenshot d. Verf.)

Abb. 2 Selbstzeugnis auf Instagram: Teigen und Ehemann John Legend mit ihrem stillgeborenen Sohn Jack, 01.10.2020 (Screenshot d. Verf.)
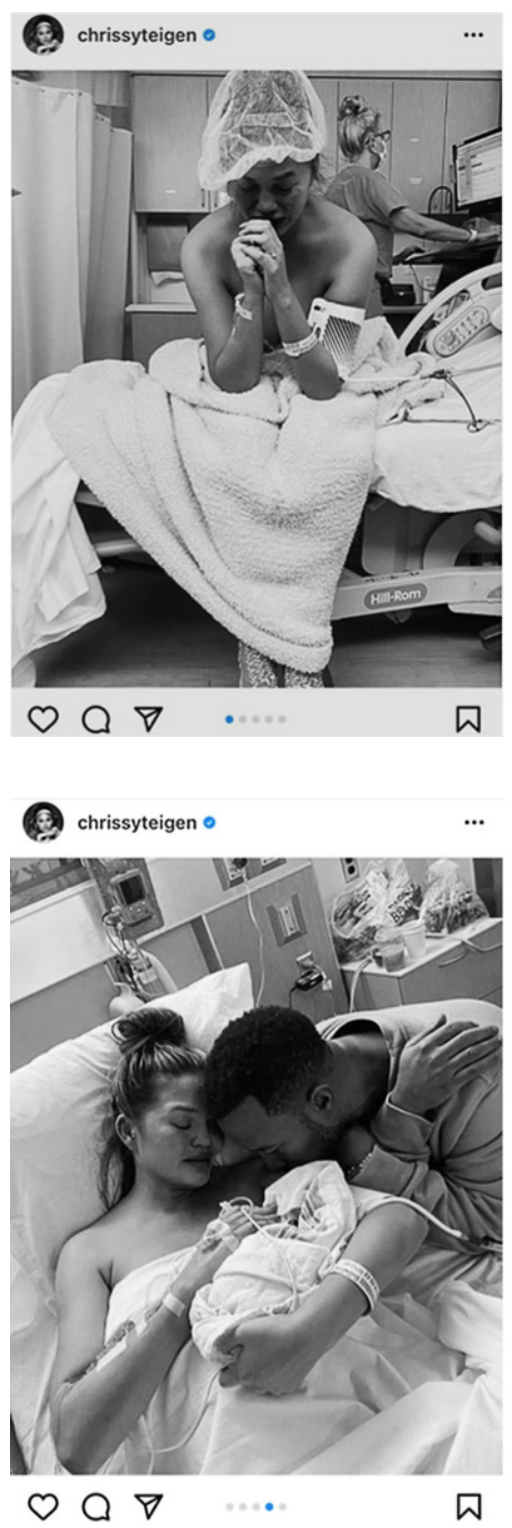

Stillgeborene (für die Niederlande siehe Peelen 2009) und Gesetzesänderungen in mehreren EU-Ländern. Innerhalb der letzten Dekade wurden u.a. in Deutschland (2013), in Belgien (2015), Österreich (2017) und in der Schweiz (2017) Personenstandsgesetze für Fehlgeborene geändert bzw. eingeführt (Schweizerische Eidgenossenschaft 2017, S. 11f.). Die Gesetzesnovellen gingen wiederum einher mit Anpassungen von Bestattungsverordnungen und, wie ich am Beispiel von Deutschland zeigen werde, neuen Routinen der Personalisierung von Fehl- und Stillgeborenen in der klinischen Geburtshilfe. 
Der Wandel geht auf das Engagement von Betroffenen zurück, die schlechte Erfahrungen mit medizinischen Routinen, einer entmachtenden Rechtslage sowie fehlender sozialer Unterstützung gemacht haben. Ein Hintergrund des Engagements ist der umstrittene Status Fehl- und Stillgeborener, der z. B. dazu führt, dass Betroffenen geburtshilfliche Maßnahmen vorenthalten oder sie mit der Vorstellung konfrontiert werden, dass ,es ja noch nicht gelebt“ habe und der Verlust entsprechend nicht ,betrauernswert" (Butler 2010, S. 57) sei. Aufgrund solcher Delegitimierungen setzen sich Aktivist*innen auch dafür ein, dass Fehl- und Stillgeburten in der Geburtshilfe und per Gesetz als ,richtige“ Geburten von ,echten“ Kindern behandelt und begleitet werden. Damit verbunden ist mitunter die Forderung, als Eltern gesehen und anerkannt zu werden. Mit Fokus auf diesem Anliegen rekonstruiere ich einen Wandel des gesellschaftlichen Diskurses und Umgangs mit Fehl- und Stillgeburten. Argumentiert wird, dass Fehl- und Stillgeburt zunehmend als Geburt (und Verlust) eines Kindes behandelt und Betroffene als (sogenannte Sternen-)Eltern anerkannt werden. Über das Engagement konnte sich ein öffentliches Bewusstsein dafür etablieren, als Betroffene ein Recht auf Trauer und Anerkennung zu haben. Diese Entwicklung bezeichne ich im Folgenden als Anerkennungswandel.

Zunächst werden das methodische Vorgehen und die Datengrundlage für die These eines Anerkennungswandels beschrieben (Abschn. 2). Es folgen begriffliche Klärungen zum Phänomen und theoretische Überlegungen, die die Ergebnisse innerhalb einer Soziologie der Schwangerschaft und Geburt verorten (Abschn. 3). In bisherigen Studien werden entweder „Idealverläufe“ von Schwangerschaft und Geburt vorausgesetzt oder die fallspezifische Komplexität disruptiver Schwangerschaften fokussiert. Mit der Rekonstruktion der (Neu-)Ordnung von kulturellen Anerkennungsverhältnissen um Fehl- und Stillgeburt füllt der Beitrag eine Forschungslücke. Der Wandel wird in drei gesellschaftlichen Bereichen - im deutschen Recht, in sozialen Medien und in der Geburtshilfe - anhand von interpretativen Daten konkretisiert (Abschn. 4). Der Beitrag schließt mit einem Fazit und weiterführenden Fragen (Abschn. 5).

\section{Empirische Rekonstruktion des Anerkennungswandels}

Empirische Grundlage dieses Beitrages sind im Rahmen meiner Dissertation zwischen 2014 und 2019 erhobene und theoretisch gesampelte Daten. Die qualitativ-rekonstruktive Studie zu Erfahrungen von Fehl- und Stillgeburt (Böcker 2022 (im Erscheinen)) wurde im forschungspragmatischen Stil der „Grounded Theory“ (Strauss 1998; Przyborski und Wohlrab-Sahr 2008) durchgeführt. Mit dem Ziel, sukzessive eine empirisch gesättigte Theorie zu entwickeln, leiteten die Ergebnisse der Datenanalyse die weitere Datenerhebung nach dem Prinzip des systematischen Vergleichens an (Strübing 2014). Ausgewertet wurden die Daten mittels hermeneutischer „Sequenzanalyse“ (Erhard und Sammet 2018; Oevermann et al. 1979). Der Datenkorpus besteht u.a. aus zwölf narrativen Interviews mit Betroffenen und Expert*innen, Beobachtungsprotokollen, öffentlichen Dokumenten, Online-Posts in Trauerforen und auf sozialen Medien sowie Vlog-Beiträgen auf YouTube. Im Ergebnis steht ein theoretisches Modell der Verlustkonstitution, das zeigt, unter welchen 
Abb. 3 Diskursives Engagement auf Twitter: Kritik am ärztlichen Umgang mit dem Fehlgeborenen und den Betroffenen, 19.05.2021 (Screenshot d. Verf.)

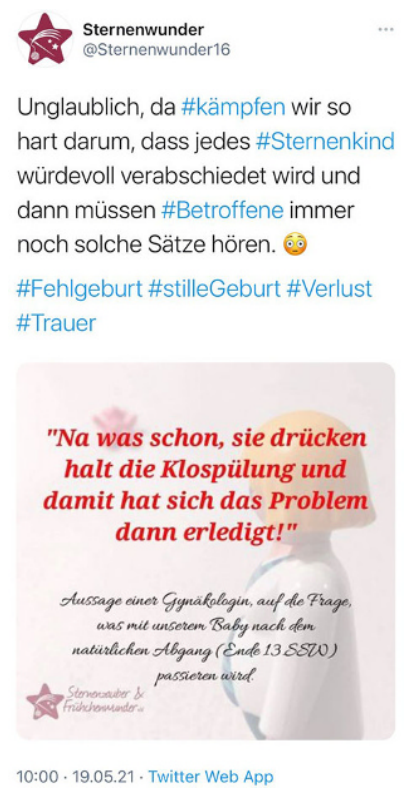

Bedingungen es aus intersubjektiver Perspektive legitim ist, nach einer Fehl- oder Stillgeburt vom Verlust eines Kindes zu sprechen (Böcker 2022 (im Erscheinen)).

Im Laufe der Forschung zeichnete sich die in diesem Beitrag im Fokus stehende gesellschaftliche Tendenz ab. Neben dem Aufkommen neuer Begriffe (und Hashtags wie \#StilleGeburt, vgl. Abb. 3) fiel z. B. auf, dass die Interviewten von Personalisierungen im klinischen Kontext berichteten, die direkt auf die Änderung des Personenstandsgesetzes (PStG) 2013 zurückgehen. Die Daten wurden in Deutschland erhoben und im Rahmen der deutschen Rechtslage analysiert, d.h. empirisch kann der Wandel nur für diesen Kontext begründet werden. Punktuell verweise ich auf vergleichbare Veränderungen in anderen Ländern und greife auf angelsächsische Studien zurück, um meine Argumentation zu stützen.

Konkret ziehe ich aus dem Datenkorpus ein YouTube-Video (in 4.2) und zwei Interviews (in 4.3) heran, anhand derer die rezenten Veränderungen in der Deutung und (professionellen Begleit-)Praxis des Fehl- und Stillgebärens besonders deutlich werden. In den ausgewählten Fällen finden Fehlgeburten zu drei verschiedenen Zeitpunkten der Schwangerschaft statt (in der 6. SSW, in der 14. SSW und in der 22. SSW). Das Video zu einer frühen Fehlgeburt in der 6. SSW wird als Versuch interpretiert, die Deutung als „Kleine Geburt“ durchzusetzen. Die Analyse zeigt somit auch exemplarisch, wie sich der Bedeutungswandel auf der Handlungsebene vollzieht. Von den Interviewten werden deutlich spätere Fehlgeburten hingegen weniger eindeutig als Kindsgeburten konstruiert, wenngleich ihnen in der geburtshilflichen Praxis etablierte (22. SSW) und ambivalente (14. SSW) Angebote der Personalisierung des Still- bzw. Fehlgeborenen gemacht worden sind. Diese Fallbeispiele stehen für einen privaten Umgang mit Fehl- bzw. Stillgeburt, der den Anliegen einer öffentlichen Anerkennungsbewegung zuwiderläuft. 


\section{Theoretische Überlegungen zur gesellschaftlichen Anerkennung von Fehl- und Stillgeburt}

\subsection{Begriffe eines umstrittenen Phänomens}

Für den Verlust des „Ungeborenen“ (Duden 2002) bzw. Schwangerschaftsverlust (vom Englischen ,pregnancy loss") gibt es zahlreiche Bezeichnungen, was bereits auf die (medizinische) Komplexität und die gesellschaftlichen Deutungskonflikte hinweist, die mit dem Phänomen verbunden sind. Auf die im Beitrag verwendeten Begriffe gehe ich kurz ein.

Juristisch ist die Unterscheidung von Lebendgeburt, Totgeburt und Fehlgeburt im PStG geregelt (Weilert 2017). Gibt es nach der Geburt ein Lebenszeichen, gilt das Kind unabhängig vom Gewicht als Lebendgeburt. Totgeborene sind sogenannte „Leibesfrüchte“ ab der 24. SSW oder einem Körpergewicht von mindestens $500 \mathrm{~g}$, bei denen sich kein Lebensmerkmal gezeigt hat (BMJV und BfJ 2021). Um eine Fehlgeburt handelt es sich, wenn die "Leibesfrucht“" weniger als $500 \mathrm{~g}$ wiegt. ${ }^{1}$ Medizinisch ist bei einer Fehlgeburt, sofern nicht operativ abgesaugt wird, ${ }^{2}$ auch von einem Abort oder natürlichen Abgang die Rede. Ein Ausdruck des im Fokus stehenden Wandels ist die Etablierung der Begriffe „Kleine Geburt“ und „Stille Geburt“ (bzw. Stillgeburt, vom Englischen ,stillbirth“), die anstelle von Fehlgeburt und Totgeburt zunehmend verwendet werden, ${ }^{3}$ sowie die öffentliche Rede von ,Sternenkindern“ und „Sterneneltern“" seit Ende der Nullerjahre. ${ }^{4}$

„Kleine Geburt“ und „Stille Geburt“ sind Alternativbegriffe, die dem Stigma des Scheiterns etwas entgegensetzen, das mit den Begriffen Fehl- und Totgeburt assoziiert wird. Statt eine Geburt zu negieren oder diese als gescheitert abzuwerten, wird mit positiver Konnotation konstatiert, dass eine Geburt stattgefunden hat. Zudem entdifferenzieren die Bezeichnungen rational-klinische und dualistische Kategorien, die nicht zu den subjektiven Erlebensweisen passen. „Stille Geburt“ beispielsweise kann sich sowohl auf späte Fehlgeburten als auch auf Totgeburten (im rechtlichen Sinne) beziehen, und „Sternenkinder“ auf alle im Rahmen einer Schwangerschaft oder Geburt verlorenen Kinder. Manchmal werden so auch im Säuglings- oder Kleinkindalter verstorbene Kinder genannt. Die zunächst bewegungsinterne Bezeichnung „Sternenkinder“" wurde von öffentlicher Seite übernommen, mittlerweile auch durch

\footnotetext{
1 Die Unterscheidung ist grob an der Grenze zur fötalen Lebensfähigkeit außerhalb der Gebärmutter orientiert.

${ }^{2}$ Häufig wird der geläufigere Begriff Ausschabung (oder Kürettage) verwendet, obwohl das Verfahren in der medizinischen Praxis durch die Methode der Absaugung abgelöst worden ist.

3 Begriffe werden uneinheitlich verwendet. Manchmal sind „,Kleine Geburt“ und „Stille Geburt“ Äquivalente der Rechtsbezeichnungen (Fehlgeburt und Totgeburt), manchmal sind mit „Kleinen Geburten“ natürliche Abgänge im Kontrast zur Absaugung gemeint. Diese unterscheiden sich von „Stillen Geburten“, bei denen das (größere) Baby vaginal geboren werden muss. Zu weiteren Differenzierungen und begrifflichen Widersprüchen siehe Böcker (2022 (im Erscheinen)).

42008 wird in Berlin-Schöneberg das Grabfeld „Garten der Sternenkinder“ eröffnet, 2009 die „Petition Sternenkinder" beim deutschen Bundestag eingereicht (siehe 4.2). In dieser Zeit etabliert sich auch der Begriff „Stille Geburt“; „Kleine Geburt“ taucht in der Deutschen Hebammenzeitschrift 2020 erstmals auf.
} 
das österreichische und das deutsche Familienministerium (BMFSFJ 2018; Bundeskanzleramt 2019).

\subsection{Personalisierung des (stillgeborenen) Fötus und Elternwerdung}

In der deutschsprachigen „Soziologie der Schwangerschaft“ (Hirschauer et al. 2014) bzw. „Soziologie der Geburt“ (Villa et al. 2011) wurden Fehl- und Totgeburten bislang vernachlässigt (vgl. Peter und Feith 2021). Seit Kurzem entstehen mikrosoziologische Studien zu Fehlgeburt und Totgeburt, die die Brüche, Paradoxien und Ambivalenzen des Schwangerschaftserlebens und der Konstruktion einer ,,pränatalen Entität“ (Völkle 2021, S. 120) freilegen (etwa Völkle und Wettmann 2021; Wettmann 2021; Böcker 2017).

Fehlgeburt, Totgeburt und der Tod eines Neugeborenen sind ambivalente Erfahrungen, die mit Stigmatisierungen und entrechteter Trauer einhergehen (Lang et al. 2011; Brierley-Jones et al. 2015). Ambivalenzen resultieren u. a. aus der „Inwändigkeit des Sterbens“ (Böcker 2017, S. 153), der ,Verstecktheit des Verlusts“ (WernerLin und Moro 2004, S. 248) und der „Liminalität“ der Schwangerschaft, nach deren abruptem Ende das Ungeborene und die nicht mehr werdende Mutter in einer Statuslosigkeit verbleiben (Kuberska et al. 2020). Der liminale Status des Stillgeborenen „between a baby and ,human tissue“" (ebd., S. 150) liegt auch dem Konflikt um Anerkennung des Verlustobjektes zugrunde. Während die einen um den Tod eines Familienmitgliedes trauern, behandeln Außenstehende das Ereignis als statistisch normalen Abgang, das Verlorene als „Zellhaufen“ (Frost et al. 2007, S. 1012).

Mit der Schwangerschaft beginnt in „westlichen“ Gesellschaften die Sozialisation des Ungeborenen, eine Phase ,,pränataler Sozialität“ (Hirschauer et al. 2014). Aufgrund von Ultraschalltechnik, Vertrauen in das medizinische System (Überlebensfähigkeit bei Frühgeburt) und medialen Darstellungen des Fötus verstehen sich Schwangere heute zumeist als werdende Eltern, sobald die Schwangerschaft bekannt ist. „They may follow the weekly physiological development of their ,baby“ with a home pregnancy manual, and each prenatal visit contributes and confirms the growing sense of the baby's realness and their growing sense of themselves as ,mothers. “" (Layne 2003, S. 17). Linda Laynes Beschreibung der wachsenden ,realness“ des Babys während der Schwangerschaft verknüpft fötale Personalität und Elternschaft. Ein solches, relationales Verständnis liegt auch diesem Beitrag zugrunde: ,[a] concept of it - whether child, baby, fetus, or embryo - is always a concept of social relations. With a child or baby, a woman is a mother, and without one, she is not; there also is no child without a mother" (Han 2019, S. 59f.). Kindliche Personalisierung und Elternwerdung bedingen sich im Fortgang der Schwangerschaft wechselseitig.

Obwohl der vorgeburtliche Bindungsaufbau durch Schwangere vonseiten der Medizin erwartet und durch Ultraschalluntersuchungen gefördert wird (Heimerl 2013; Sänger 2020), wird nach einem Verlust mitunter so agiert, als habe es keine Personalisierung gegeben. Die rationalisierende Terminologie (etwa die Rede von Schwangerschaftsgewebe) macht, so die Perspektive betroffener Eltern, ihr Kind zur Sache und führt zu zusätzlichen Gefühlen der Entmachtung und des Alleingelassenseins (vgl. Frost et al. 2007, S. 1012). Der von Betroffenen wahrgenommene ,lack of 
Abb. 4 Engagement auf Twitter: handgenähte Babykleidung im Kontrast zur Nierenschale, die für einen würdelosen Umgang mit Föten in Kliniken steht, 10.05.2021 (Screenshot d. Verf.)
Sternenwunder

@Sternenwunder16

Müll oder würdevoller Abschied?

\#Sternenkind \#Sternenmama

\#Sternenpapa \#Fehlgeburt

\#StilleGeburt \#Ehrenamt

\#weilhelfenhilft

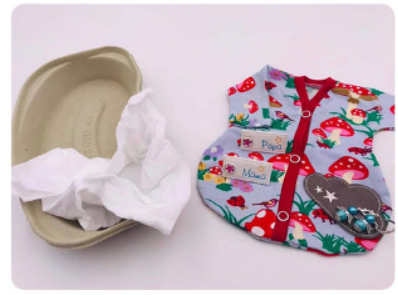

17:05 1 10.05.21 $\cdot$ Twitter Web App

appreciation“ (Cecil 1994, S. 21) durch medizinisches Personal ist seit Jahrzehnten und noch immer Gegenstand vehementer Kritik. In den sozialen Medien ist die Entrechtung, über die Bedeutung und Behandlung des eigenen fehl- oder stillgeborenen Fötus zu bestimmen, die Beweggrund für das Engagement der 1980er war, inzwischen eine Fokussierungsmetapher im sternenelterlichen Diskurs (siehe Tweet in Abb. 3). Zentral entzündet sich der Konflikt am Umgang mit dem Fötus nach der Entbindung, der sich mit dem Kontrast von Lagerung in einer kalten Nierenschale und liebevoller Einkleidung des kindlichen Leichnams auf den Punkt bringen lässt (Abb. 4). Die kollektiven Bestrebungen, dem ambivalenten Ereignis die soziale Bedeutung eines Kindesverlustes zu verleihen, stehen in diesem Beitrag im Vordergrund.

\subsection{Anerkennung von Verlust und (Sternen-)Elternschaft}

Die Lage, in der sich Personen nach einer Fehlgeburt bzw. Stillgeburt wiederfinden, lässt sich mithilfe des theoretischen Konzeptes der „entrechteten Trauer“ nach einem „nicht anerkannten Verlust“ (Doka 2002) fassen. In den 1980er-Jahren von Kenneth Doka entwickelt, ist das soziologische Konzept mittlerweile in der professionellen Trauerbegleitung etabliert (Thompson und Doka 2017, S. 177). Entrechtet werden Trauernde beispielsweise nach Verlusten in illegitimen oder von der Norm abweichenden Beziehungen oder durch stigmatisierte Todesumstände, wie Suizid oder HIV/AIDS (Werner-Lin und Moro 2004). Eine andere Ursache der Entrechtung ist, wenn der Verlust nicht gesehen, die verlorene „Sache“ als wertlos betrachtet und die Art oder Intensität der Trauerreaktion entsprechend negativ sanktioniert wird (vgl. Thompson und Doka 2017, S. 178-181). Fehl- und Stillgeburten sind aus Perspektive der Betroffenen oft solche nicht anerkannten Verluste.

Angelsächsische Arbeiten zum Umgang mit Fehlgeburt, Totgeburt und dem Tod Neugeborener haben die Situation in den Kliniken und die fehlende kulturelle Sichtbarkeit des Verlusts bereits in den 1980er-Jahren problematisiert (Lovell 1983; Ran- 
do 1986). Noch vor 15 Jahren konstatierten Julia Frost et al. in ihrer Studie mit 79 Frauen aus England, die im ersten Trimester eine Fehlgeburt erlitten hatten: „There is currently no public way in which the loss of a miscarried baby can be marked, no consoling rituals or commonly accepted ways of coming to terms with the bereavement" (Frost et al. 2007, S. 1018). Seit diesen Pionierstudien hat, so meine These, ein anhaltender Wandel von Anerkennungsverhältnissen und -routinen nach Schwangerschaftsverlusten in Geburtshilfe und Gesellschaft eingesetzt, die den Ausdruck von Trauer um das ungeborene Kind und entsprechende Abschiedsrituale unterstützen und legitimieren.

Heuristisch unterscheide ich zwei Phänomene von Anerkennung, die im Zuge des Wandels Zuwachs erfahren (haben): private Praktiken der Personalisierung und ein öffentlicher Diskurs um Anerkennung. Zum einen sind Praktiken institutionalisiert worden, denen eine Deutung des Fehl- bzw. Stillgeborenen als Kind und soziale Person zugrunde liegt. Diese (diskursiven) Praktiken sind „Anerkennungsakte“ (Graumann 2011, S. 387), bei denen die Anerkennung, die dadurch zum Ausdruck gebracht und beobachtbar wird, nicht bewusst sein muss. Zum Beispiel bringen Handelnde nach der Geburt das Stillgeborene als verstorbene Person hervor, indem sie es anziehen und fotografieren (Völkle 2021). Diese Praktiken adressieren symbolisch-performativ die menschliche Personalität und Individualität des Stillgeborenen und sind nicht politisch intendiert. Hebammen helfen Eltern so etwa, ihr stillgeborenes Kind zu verabschieden, den Verlust zu begreifen, und erleichtern damit die Trauerarbeit (von der Hude und Glückselig 2014). Diese Personalisierungen werden im familiären Kreis vollzogen und sind weit über zivilgesellschaftliche Zusammenschlüsse hinaus in der Geburtshilfe etabliert und verbreitet.

Die zweite Tendenz trägt hingegen politisch-normative Implikationen: Zivilgesellschaftlichen Akteur*innen geht es um die öffentliche Anerkennung von Fehl- und Stillgeborenen als menschliche Personen sowie, daran geknüpft, von Betroffenen als Eltern. Dieses Anerkennungsphänomen beinhaltet einen Diskurs um Anerkennung, welche, so die Perspektive der Akteur*innen, bislang unzureichend ist. Ein pointiertes Beispiel bietet der Ratgeber „Stille Geburten sind auch Geburten und Sterneneltern sind auch Eltern“ von Corinna Hansen-Krewer (2021). Ihren persönlichen Erfahrungsbericht verbindet Hansen-Krewer mit dem Anliegen, Anerkennung für ,Sterneneltern“ zu generieren:

Vor allem aber handelt es [das Buch, Anm. d. Verf.] davon, wie die Eltern von viel zu früh verstorbenen Kindern endlich die würdevolle Behandlung erhalten, die sie brauchen und auch verdienen. Stellen Sie sich einmal vor, dass Stille und Kleine Geburten die gleiche Wertigkeit wie Lebendgeburten hätten. Was wäre, wenn die Gesellschaft anerkennen würde, dass Sterneneltern auch Eltern sind, die Bedürfnisse und Emotionen haben? (Klappentext)

Es sind nicht (nur) die „viel zu früh verstorbenen Kinder“ selbst, denen eine „würdevolle Behandlung“, etwa Bestattung, verwehrt wird. Vielmehr ist der Vorwurf, dass die Gesellschaft, indem sie Fehl- und Stillgeburten von Lebendgeburten unterscheidet, Sterneneltern ihre Elternschaft und Würde vorenthält und ihnen somit verweigert, was „sie brauchen und auch verdienen“ (Herv. d. Verf.). In diesem Diskurs geht es also auch um gesellschaftliche Anerkennung einer elterlichen Leistung. 
Die Konflikte stehen somit im Kontext gesellschaftlicher Anerkennungsverhältnisse, in denen, so Judith Butler, soziale Positionen zugeordnet werden und verhandelt wird, welche Leben als „betrauerbar“ (Butler 2010, S. 9) gelten. Betroffene fordern das Recht, selbst darüber zu bestimmen, ob Fehl- und Stillgeborene betrauerbar sind, und transformieren damit ihre Subjektpositionen in Richtung legitimer Elternschaft.

\section{Anerkennungswandel}

\subsection{Anstoß des Wandels: Zivilgesellschaftliches Engagement}

Seit den 1980er-Jahren setzen sich Selbsthilfe- und Eltern-Initiativen für die Unterstützung von Personen ein, die von Fehlgeburt, Totgeburt oder dem Tod ihres Neugeborenen betroffen sind. Sie fordern „humanere“ klinische Routinen und ein umfassendes Recht auf Bestattung und versuchen, gesellschaftliches Bewusstsein für die Probleme zu schaffen, die aus dem unsensiblen Umgang mit Betroffenen resultieren. Die zu jener Zeit vorherrschenden klinischen Routinen und fehlenden Rechtsansprüche führten nicht bei allen Betroffenen zu dem Gefühl, entrechtet worden zu sein. Vielmehr stießen einzelne Pionier*innen, die das zunächst betraf, die Etablierung einer alternativen Deutungsordnung an, vor deren Hintergrund bestimmte Erfahrungen überhaupt erst als Entrechtung interpretier- und skandalisierbar wurden.

Die erste Elterninitiative zum Tod eines Kindes in Deutschland gründeten Barbara Künzer-Riebel und Regine Schreier im Jahr 1983. Die „Initiative Regenbogen“ hatte zum Ziel, Eltern, die vor, während oder nach der Geburt ein Kind verloren haben, Unterstützung anzubieten und Ansprechpersonen zu vermitteln (Lutz und Künzer-Riebel 1989). Seit dem Zusammenschluss mit der Selbsthilfegruppe „Glücklose Schwangerschaft“ 1987 agiert die Initiative deutschlandweit als „Initiative REGENBOGEN - Glücklose Schwangerschaft e.V." und setzt sich u.a. für Anlaufstellen und Ansprechpersonen, Veränderungen in Kliniken, die Einrichtung separater Gräberfelder und Gesetzesänderungen ein (Initiative Regenbogen e. V. 2021). Bereits 1988 engagierte sie sich erfolgreich für eine Änderung des PStG (seit 1994 werden totgeborene Kinder ab einem Geburtsgewicht von $500 \mathrm{~g}$ personenstandsrechtlich erfasst) und das Recht auf Bestattung. Und sie artikulierte bereits zu dieser Zeit das Problem entrechtender Kommentare im alltäglichen Umgang und forderte das „Anerkennen des Babys als Individuum und es nicht mit Worten wie ,Das ist ja nicht so schlimm, sie werden noch andere Kinder haben können' abzuwerten“ (Lutz und Künzer-Riebel 1989, S. 96). Künzer-Riebel wurde nach der Geburt ihr sterbendes Kind vorenthalten, weshalb die von ihr ins Leben gerufene Initiative besonders dem Anliegen verpflichtet war, dass Betroffene ihr Kind sehen und über dessen Verbleib entscheiden dürfen.

Die Initiativen wurden und werden von Personen getragen, die selbst einen Verlust erlitten haben. Das Engagement fungiert mithin auch als Trauerarbeit und Initiativen existieren nur, solange sich deren Mitglieder mit dem eigenen Verlust auseinandersetzen wollen. Viele Initiativen sind lokal organisiert und setzen sich für individuelle 
Unterstützung und die Verbesserung in verschiedenen Bereichen ein. ${ }^{5}$ So gibt es beispielsweise Rückbildungskurse für ,,verwaiste Mütter“6, Vernetzungstreffen und Trauercafés für „Sterneneltern“ sowie Näh- und Bastelgruppen, die „Erinnerungsboxen" mit Kleidung und Accessoires für die Kliniken herstellen. International stehen freiwillige Fotograf*innen auf Abruf zur Verfügung, um in den Kliniken „Sternenkinder" zu fotografieren (Dein-Sternenkind Stiftung 2021). Online engagieren sich Einzelakteur*innen für die Verbreitung von Informationen und gesellschaftliche Normalisierung. Ein Beispiel ist die 2017 von der Journalistin Julia Stelzner „,[g] egen ein Tabuthema“ gegründete Webseite „Das Ende vom Anfang“, auf der Erfahrungsgeschichten von Fehlgeburten gesammelt werden (DEVA 2021). Das Ziel, gesellschaftliches Bewusstsein zu schaffen, mag in den Initiativen nur eines unter mehreren sein und für manche Ehrenamtliche keine Relevanz besitzen. Einige Initiativen, wie einst die überregionale „Petition Sternenkinder“ zur Änderung des PStG für Fehlgeborene (ausführlich Abschn. 4.2), kämpfen hingegen ausdrücklich für die offizielle Anerkennung von „Sterneneltern“.

Im Folgenden plausibilisiere ich die These, dass ein Anerkennungswandel stattgefunden hat, anhand von Veränderungen in drei Bereichen. Erstens wurde durch die Änderung des PStG ein symbolisch-institutioneller Wandel angestoßen, der Anerkennung für „Sterneneltern“ generiert (Recht, 4.2). Zweitens sind Fehl- und Stillgeburten durch Medialisierung öffentlich sichtbarer und es ist legitimer geworden, die vormals privaten Erfahrungen zu teilen. Die digital verfügbaren Selbstzeugnisse transformieren wiederum den Diskurs um den gesellschaftlichen Umgang (Soziale Medien, 4.3). Drittens sind in der geburtshilflichen Praxis professionelle Routinen etabliert, Stillgeborene zu personalisieren (Geburtshilfe, 4.4).

\subsection{Die rechtliche Anerkennung von ,Sterneneltern“6}

Im Mai 2013 wurde in Deutschland das PStG dahingehend geändert, dass Eltern für ihre Fehlgeborenen eine Bestattung und eine standesamtliche Beurkundung veranlassen können (Weilert 2017). Zuvor wurden Fehlgeborene meist ohne weitere Dokumentation mit dem Klinikabfall entsorgt. Bestattung und Beurkundung der Fehlgeburt sind freiwillig. Die standesamtliche „Bescheinigung“ der Fehlgeburt, in der der vorgesehene Name des fehlgeborenen Kindes eingetragen werden kann, sieht aus wie eine Geburtsurkunde und ist ihr symbolisches Substitut (Böcker 2021); Fehlgeburten werden, anders als Totgeburten, nicht in den Personenstandsregistern dokumentiert. Seit der Novelle besteht in den meisten Bundesländern zudem eine Pflicht für behandelnde Einrichtungen, Betroffene zu informieren, dass sie selbst eine Bestattung veranlassen können, und, sofern Eltern sich dagegen entscheiden, „Fehlgeburten und Ungeborene [...] unter würdigen Bedingungen zu sammeln und zu bestatten“ ( $\$ 30$ Abs. 3 S. 2 BestattG). Da Bestattung Sache der Bundesländer

\footnotetext{
5 Eine umfangreiche Link-Sammlung zu Initiativen im In- und Ausland findet sich auf der Webseite der Initiative REGENBOGEN - Glücklose Schwangerschaft e. V.

6 Vor dem Aufkommen von „Sterneneltern“ gebräuchliche Selbstbezeichnung von Personen, die ein Kind verloren haben. Begriff findet sich auch im Namen des Bundesverbandes Verwaiste Eltern und trauernde Geschwister in Deutschland e. V. (VEID).
} 
ist, variieren die konkreten Regelungen innerhalb Deutschlands bisweilen erheblich (Gscheidel und Schwarz 2021). Die meisten Kliniken sammeln die Fehlgeborenen und organisieren in regelmäßigen Abständen Sammelurnenbestattungen, die manchmal von einer Trauerfeier, z. B. durch die Klinik-Seelsorge, begleitet sind. Betroffene werden vorab über die Beisetzung informiert bzw. zur Trauerfeier eingeladen.

Die Änderung des PStG ist eine zentrale Referenz innerhalb des Diskurses über den zu verändernden Umgang mit Fehl- und Totgeburten. So heißt es in einem Erfahrungsbericht: „Erst durch eine Elterninitiative wurde im Jahr 2013 das Personenstandsrecht für alle totgeborenen Kinder unabhängig von ihrem Geburtsgewicht geändert. Nach $\S 31$ Abs. 3 der Verordnung des Personenstandsgesetzes besteht ab 2013 für alle Mütter und Väter zumindest die Möglichkeit, ihr totes Kind als Person beurkunden $\mathrm{zu}$ lassen. Rechtlich hat das Kind damit existiert und die Eltern sind als Eltern anerkannt.“ (Faust 2021, S. 115). Die betroffene Autorin Julia Faust erzählt in ihrem Text vom Tod ihres Kindes Jahre vor der Gesetzesänderung. Deutlich wird im Zitat die Statustransformation, die „,das Kind“ - und damit nachträglich ihr stillgeborenes Kind - mit der Gesetzesänderung vollzogen hat. Die Eintragung stellt eine nachträgliche Anerkennung dar, die auch eine Wiedergutmachung für erfahrene Entrechtungen durch medizinisches Personal und das soziale Umfeld ist.

In Österreich besteht seit April 2017 eine vergleichbare Möglichkeit zur Beurkundung einer Fehlgeburt (Bundeskanzleramt 2019, S. 13). Wie in Deutschland kann die Urkunde auch im Nachhinein ohne zeitliche Begrenzung ausgestellt werden, sofern eine medizinische Bestätigung über die Fehlgeburt vorgelegt wird. In den Bundesländern Kärnten, Burgenland und Tirol besteht ein Recht auf Bestattung von Fehlgeborenen, in Wien, Niederösterreich, Oberösterreich, Salzburg, der Steiermark und Vorarlberg eine Bestattungspflicht (ebd., S. 9). Während der Umgang mit den sterblichen Überresten durch die behandelnden Einrichtungen in den deutschen Bundesländern uneinheitlich geregelt ist und je nach Klinik variiert, heißt es in der vom österreichischen Bundeskanzleramt (ebd., S. 10) herausgegebenen Broschüre unmissverständlich: „Ein markiertes Grab gibt den Betroffenen einen [...] Ort, an dem Trauer ausgelebt werden kann. Wenn das Kind nicht von den Eltern beerdigt wird, muss das Krankenhaus dafür Sorge tragen“. Von offizieller Seite steht hier nicht zur Debatte, dass es sich bei den Fehlgeborenen um Kinder handelt, denen eine würdige Bestattung zusteht.

In beiden Ländern wurden die Gesetzesänderungen durch Initiativen betroffener Eltern angestoßen. In Österreich initiierte 2012 Anita Ogris die Online-Petition „Abschaffung der 500-Gramm-Grenze bei Fehlgeburten und freiwillige Eintragung aller Kinder ins Personenstandsregister" (Verein Pusteblume 2014). Die entsprechende Gesetzesänderung trat 2017 in Kraft. In Deutschland hatten 2009 Barbara und Marion Martin die „Petition Sternenkinder“ ins Leben gerufen. Sie hatten drei Kinder verloren, die mit einem jeweiligen Geburtsgewicht unter $1000 \mathrm{~g}$ weder beurkundet noch bestattet werden konnten. Das Ehepaar hat seinen Weg bis zur erfolgreichen Gesetzesänderung auf einer Webseite dokumentiert, und darüber ein Buch geschrieben (Martin und Martin 2014). Die Gesetzesänderung folgt einem Narrativ vormals entrechteter Elternschaft, das die Martins öffentlich bekannt gemacht haben und für das sie selbst idealtypisch stehen. Das Narrativ und die entsprechend geänderten 
Regularien sind allerdings, wie gezeigt wird (siehe 4.4), nicht für alle Betroffenen passend.

\subsection{Von Fehlgeburt zur ,Kleinen Geburt": Mediale Sichtbarkeit und Transformation}

Digitale Kommunikation stellte einen zentralen Antrieb des kulturellen Wandels dar, nicht nur weil sich durch sie die Reichweite für Initiativen vergrößert hat. ${ }^{7}$ Vor allem trugen Vergemeinschaftung und Selbsthilfe Betroffener im Internet zur Etablierung eines kollektiven Selbstverständnisses individuell betroffener „Sterneneltern“ bei. ${ }^{8}$ In einem Trauer-Forum für ,verwaiste Eltern“ lautet beispielsweise ein Eintrag von 2013:

WIR SIND ELTERN. Das muss man sich immer wieder klar machen. Und Gott sei Dank behandeln uns unsere Nachbarn, die alle Kinder haben, auch so. Zum Muttertag habe ich dieses Jahr so viel bekommen.

Der Post bringt den Widerspruch zwischen dem foreninternen Konsens und der sozialen Wirklichkeit außerhalb des Forums zum Ausdruck: Die Betonung der Aussage „Wir sind Eltern“ versucht, dieser Wahrheitsgehalt und Sichtbarkeit zu verleihen und offenbart damit zugleich performativ, dass sie (noch) nicht Common Sense ist. Auch in der Belegerzählung für die Realität, Mutter zu sein, nämlich zum Muttertag bedacht und damit als Mutter wahrgenommen worden zu sein - unterscheidet die Sprechende sich von den Nachbar*innen, ,die alle Kinder haben“, und aktualisiert eine gegenteilige Position, nämlich kein Kind zu haben.

Die Rede von „Sternenkindern“ etablierte abseits des gesellschaftlichen Mainstreams einen legitimen Status für diese besondere und unsichtbare Erfahrung, Eltern ohne Kind zu sein. Rafanell und Sawicka (2020, S. 87f.) konzeptualisieren anhand von Analysen polnischer Online-Foren „Sternenelternschaft“ (,Angels' Motherhood“) auch als kollektive Errungenschaft der Online-Community: „By establishing the reality of the unborn as an Angel baby with an ontologically equal status to the earth children, this community redefines motherhood in ways that better suit their [...] experience as mothers [...]. According to this emerging reframing, women who have suffered a perinatal loss are now not only able, but also entitled, to think of themselves as mothers." In den Online-Foren entwickelte sich ein suböffentliches Forum, das zum einen den individuellen Leidensgeschichten einen geteilten Interpretationsrahmen verschaffte. Hier wurden Fehl- und Stillgeborene als verstorbene Kinder behandelt, deren Tod bedeutsam und betrauernswert ist. Forenmitglieder eigneten sich hier eine Identität als ,,Sterneneltern“ an, die ihnen einen Status und über die Community wechselseitige Anerkennung gewährte. Zum anderen verhalfen die in den Foren ausgelösten Diskurse, z. B. über das medizinische Deutungsmonopol

\footnotetext{
7 Die „Petition Sternenkinder“ war beispielsweise eine Online-Petition, die über 40.000 Personen unterzeichneten.

8 Online-Formen des Austausches und der Unterstützung nach Kindsverlust sind anfänglich aus lokalen Selbsthilfeorganisationen und Vereinen hervorgegangen (zur US-amerikanischen Entwicklung seit den 1970er-Jahren siehe Carlson et al. 2012).
} 
und die kommunikative Tabuisierung, vormals abweichenden Deutungen zu zunehmender Legitimität, bis sie schließlich in rechtlichen Normen institutionalisiert wurden.

Mit den sozialen Medien sind neben diese kollektiven Formen digitaler Selbsthilfe individuelle Darstellungen von Fehl- und Stillgeburt getreten. Unter dem Aspekt einer Virtualisierung des Totengedenkens sind digitale Friedhöfe, Gedenkseiten und Blogs, die zum Gedenken an ein verstorbenes Kind kreiert bzw. besucht werden, gut erforscht (exemplarisch Sani et al. 2021). Neu sind inszenierte Selbstthematisierungen von Akteur*innen, die über ihre persönlichen Kanäle, etwa via Facebook oder Instagram, ihr privates Leben teilen.

Exemplarisch analysiere ich im Folgenden ein YouTube-Video, in dem eine Vloggerin ihre frühe Fehlgeburt dokumentiert und als Geburt inszeniert, auch um öffentlich Sichtbarkeit für das mit dem Verlust verbundene Leid zu schaffen. Ich interpretiere den Fall unter der Prämisse, dass „Praktiken visueller Argumentation und Selbstdarstellung in internetgestützten Publikations- und Kommunikationsforen dazu beitragen, neue Formen von Öffentlichkeit herzustellen und Wissensbestände für eine Transformation zu öffnen“ (Traue und Schünzel 2014, S. 123). Als zugleich Ausdruck und Motor des Wandels steht er für eine neue Praxis, frühe Fehlgeburten als „Kleine Geburten“ zu verstehen, für ,unpolitisch“ motivierten Online-Aktivismus, der zur diskursiven Anerkennung von Betroffenen als (Sternen-)Mütter beiträgt sowie für eine neue Sichtbarkeit von Fehl- und Stillgeburtserfahrungen in sozialen Medien.

In ihrem Video dokumentiert Nina ihre Fehlgeburt in der 6. SSW, die ihr zum Beginn der Videodokumentation noch bevorsteht. Es ist ihre dritte Fehlgeburt. In der Rolle der Stellvertreterin, die im Namen der und für die ,vielen Frauen, die da durchmüssen“ und die ,überhaupt nicht wissen, was auf sie zukommt“, möchte Nina „wirklich mal zeigen, wie es ist, was einen erwartet“. Das Anliegen ist ein doppeltes: Einerseits richtet sich ihr Video gegen die Gefühlsnorm, eine Fehlgeburt sei nicht lang zu betrauern. ${ }^{9}$ Andererseits sucht sie ihren Verlust festzuhalten und inszeniert die Fehlgeburt als Geburt ihres Babys, um es „realer“ werden zu lassen (vgl. Layne 2003).

Nina berichtet zunächst von der Ultraschall-Untersuchung, in der die Gynäkologin feststellt, dass der Embryo sehr klein und kein Herzschlag darstellbar ist. In diesem Augenblick habe sie gewusst, dass sie eine Fehlgeburt erleiden und ihr Kind verlieren wird. Sie zeigt fünf (!) positive Schwangerschaftstests und zwei Ultraschallfotos. Mit wenigen Mitteln ruft Nina ein „Baby“ auf, dessen Geburt sie im weiteren Video inszeniert. Offenkundig wird hier die vielfach beschriebene Bedeutung medizintechnischer Medien, mittels derer Vorstellungen von pränatalem Leben, fötaler Personalität und Individualität entstehen und erzeugt werden (u.a. Petchesky 1987; Franklin 1991; Köhne 2018; Sänger 2020). Das sich über mehrere Tage hinziehende Fehlgebären selbst ist im Video nicht zu sehen; Nina greift dafür auf

\footnotetext{
9 Am Ende des Videos zitiert sie Ratschläge von Außenstehenden, wie über die Fehlgeburt hinwegzukommen sei („Ablenken“, ,mit Freunden Treffen“). Dies sei einer Unwissenheit geschuldet, weshalb sie die Realität einer natürlichen Fehlgeburt zeige. Nina setzt der dominanten Trauernorm ihr Erfahrungswissen entgegen.
} 
symbolische Artefakte - wie die Ultraschallfotos und in einem Becher gesammeltes Blut - zurück. Deutlich wird, etwa an Nahaufnahmen, in denen sie geronnenes Blut und Schleimhäute zwischen den Fingern zerreibt, dass sie den Embryo bzw. ihr Kind sucht.

Der Erfahrungsbericht hat Analogien zu einer ,normalen“ Geburtserzählung, etwa wenn die nachlassende Blutung nach einigen Tagen wie eine Wehenschwäche unter der Geburt problematisiert wird:

Ich hatte eine sehr ruhige Nacht wieder, leider habe ich nicht mehr genug geblutet, was schlecht ist, denn es soll ja alles rauskommen [...]. Ich habe heute morgen Himbeerblättertee getrunken, der hilft nämlich bei Geburten und da das hier eine kleine Geburt ist, ähm, habe ich davon erstmal eine Tasse getrunken in der Hoffnung, dass die leichten Wehenkrämpfe wieder zunehmen.

Nina stellt sich als verantwortungsvolle Mutter dar, die schon an wehenfördernden Himbeerblättertee gedacht hat, und betont, dass es sich um eine „kleine Geburt“ handelt, was die Existenz eines Kindes und einer Beziehung zu ihm voraussetzt. Die Betonung selbst weist dabei zugleich darauf hin, dass diese Deutung nicht selbstverständlich ist. Den dezidierten Moment, an dem der Embryo abgeht, kann Nina nicht wahrnehmen und dokumentieren. Nach einem erneuten Arztbesuch berichtet sie: „Die Frucht ist schon raus, also mein Kind habe ich schon verloren.“ Es ist ein zweites Mal die Ultraschall-Untersuchung, die den Verlust des Kindes zum Fakt gemacht hat.

Das Beispiel zeigt, dass die Erfahrung, durch Fehlgeburt ein Baby zu verlieren vermittelt über Ultraschalldiagnostik und Social Media -, vor oder parallel zum eigentlichen Abgang erlebt und dargestellt werden kann. Der elterliche Verlust und das leiblich gebundene Fehlgeburtserleben sind nur lose gekoppelt. Zugleich fungieren Artefakte der Schwangerschaft hier als „Evidenz“ für den Verlust des leiblichen Kindes und damit als Grundlage für die Konstruktion leiblicher Elternschaft. Dieses als Social-Media-Beitrag inszenierte Doing Kleine Geburt ist nur möglich, weil Nina bereits zum dritten Mal eine Fehlgeburt erleidet und über eine zweifelsfreie Deutung der Fehlgeburt als Kindsgeburt verfügt.

Mit dem Video kann sie erstens rückwirkend ihre vorherigen Verluste sowie damit verbundene Schmerzen und Einschränkungen gegenüber Dritten sichtbar machen und ,beweisen“. Zweitens vollzieht sie eine Umdeutung von der stigmatisierten Fehlgeburt - schon der Begriff verneint eine Geburt - zur Erfahrung einer ,natürlichen“ Geburt, die sie zur Mutter macht (zum Gebären als Leistung siehe Völkle und Muthmann 2019). Diese positiv besetzte Erfahrung setzt einen natürlichen Abgang voraus - und dieser wiederum das Wissen, dass eine diagnostizierte Fehlgeburt nicht immer abgesaugt werden muss. Nina bietet damit einen alternativen und selbstbestimmten Umgang mit Fehlgeburt, die nicht, wie es medizinische Routine ist, abgesaugt wird, sondern als „Kleine Geburt“ erfahren und mit der Community geteilt werden kann. Zugleich trägt sie dazu bei, die vor allem mütterliche Leistung leiblicher Reproduktionsarbeit sichtbar zu machen. 


\subsection{Etablierung der Personalisierung von Stillgeborenen in der Geburtshilfe}

Ein dritter Bereich, in dem der Anerkennungswandel beobachtbar ist, ist die Geburtshilfe. Vor, während und nach einer Fehl- oder Stillgeburt sind v. a. Erstbetroffene auf professionelle Deutungsangebote angewiesen. Bei spontanen Abgängen vermitteln Hebammen, Ärzt*innen und Klinikangestellte symbolisch oder explizit, wie die Situation zu verstehen ist (Geburt, Abgang, medizinische Routine). Steht eine Diagnose am Anfang, helfen sie bei der Planung der palliativen oder stillen Geburt. Bei drohenden Geburten, wenn noch nicht feststeht, ob das Ungeborene oder Frühgeborene zu retten ist, sind Betroffene einer krisenhaften Ungewissheit ausgesetzt, die sie in Interaktion mit Ärzt*innen aufzulösen versuchen (Peter 2013). Und Professionelle beeinflussen die Entscheidung, was mit dem fötalen Körper bzw. Schwangerschaftsgewebe nach der Entbindung passieren soll (Entsorgung, Beisetzung).

In vielen ,westlichen“ Ländern war es bis in die 1970er-Jahre üblich, dass selbst voll ausgetragene totgeborene Babys mit dem Klinikabfall entfernt wurden, ohne dass Eltern es zu Gesicht bekamen (Warland und Davis 2011). Noch 1989 kritisieren Lutz und Künzer-Riebel diese Praxis und forderten, neben geburtsbegleitenden Maßnahmen, elterliche Selbstbestimmung in Bezug auf den Umgang mit sterblichen Überresten.

Heutzutage gilt innerhalb der Geburtshilfe die eindeutige Empfehlung, Eltern nach Stillgeburt und Perinataltod bei der Familienwerdung und Abschiednahme zu unterstützen und sie zu verschiedenen Zeitpunkten des Prozesses zu ermutigen, ihr Kind anzuschauen (von der Hude und Glückselig 2014; Limbo 2012). Entsprechende Praktiken der Familialisierung sind in der letzten Dekade in der (Palliative-)Care-Forschung in den Fokus gerückt (exemplarisch Côté-Arsenault und Kavanaugh 2018). Studien betonen u. a. die Bedeutung von Fotografien des Babys, die nach der Geburt aufgenommen werden, und anderen Erinnerungsstücken, wie Fußabdrücken oder Kuscheltieren (Layne 2000; Ramirez et al. 2019). Diese „linking objects“ (Wheeler 1999, S. 289) stellen eine Möglichkeit für die Trauernden dar, eine Verbindung mit dem verstorbenen Kind aufrechtzuerhalten, und können gegenüber Dritten später als „Existenzbeweise“ fungieren.

Für eine geburtshilfliche Begleitung als Geburt und Abschied eines Kindes steht der Fall meiner Interviewpartnerin Rhea, die in der 22. SSW eine späte Fehlgeburt erleidet. Sie verbringt zwei Tage in aufwühlender Unwissenheit im Krankenhaus. Als die Fruchtblase platzt, ist klar, dass die Geburt nicht aufzuhalten ist und das Ungeborene nicht überleben wird. Mit dieser Gewissheit kommt Rhea in den Kreißsaal und in ,die guten Hände“ der Hebamme und damit zur Ruhe, nun hat sie, wie sie sagt, ,alle Zeit der Welt“. Die Hebamme sei ein „totaler Glücksfall“ gewesen, da sie mit ihr und ihrem Partner unter der Geburt nächste Schritte und Entscheidungen bespricht. Die Hebamme sorgt auch für die soziale Geburt des Kindes vor der physischen Entbindung. Erst als Rhea sie zitiert, spricht sie selbst von einem Kind: Die Hebamme habe gefragt, ob sie „das Kind denn hinterher sehen wollen“, erklärt, dass , das Kind ... nicht überleben kann“ und dass es keinen Grund gebe, Schmerzen ohne Schmerzmittel auszuhalten, ,wenn es darum geht, ein Kind, das nicht lebt, zur Welt zu bringen“. Nach der Geburt stellt sie sogleich einen körperlichen Kontakt zwischen Rhea und dem Stillgeborenen her, indem sie es ihr - wie bei jeder anderen 
Geburt - direkt auf die Brust legt. Die Hebamme trägt zu einem „kleinen Mikrokosmos" bei, indem sie dem Paar Zeit gibt, sich zu verabschieden und das Baby so lange auf dem Arm zu halten, bis es bereit ist, das Kind wortwörtlich loszulassen.

Bis auf intensiven Körperkontakt nehmen Rhea und ihr Partner keine weitere Personalisierung vor. Sie wissen, dass sie ,ihn“ zu einem späteren Zeitpunkt noch einmal anschauen können, aber das sei etwas, das sie „gar nicht nochmal brauchen“. Sie lehnen auch das „Angebot“ zu einem Foto ab (,das wollten wir aber auf jeden Fall auch nicht"“) und verzichten auf die Vergabe eines Namens, eine eigenständige Beisetzung und die Teilnahme an der Trauerfeier der Klinik. Schließlich berichtet Rhea von einem Telefonat mit einer Mitarbeiterin der Klinik, einige Wochen nach der stillen Geburt, die sich nach ihrem Befinden erkundigt - ein „Hilfsangebot“, das sie lieber ablehnt. In diesem Kontext erwähnt Rhea pejorativ das „Sternchengrab, nennen die das“, das sie nie besucht hat. Im gesamten Interview grenzt sie sich von Zuschreibungen der (Sternen-)Mutterschaft und einer Betroffenheit ab.

Auch wenn das Paar die Angebote ablehnt, bezieht sich Rhea positiv auf sie: Es sind Möglichkeiten, keine Zwänge. Stets entscheiden sie selbst, was sie „brauchen“ und „wollen“. Professionelle und Betroffene handeln hier im Laufe des Prozesses immer wieder aus, welche Formen der Personalisierung gewollt sind.

Die ethnografische Einzelfallstudie von Laura Völkle zeigt, dass die professionelle Hebammenpraxis an der Potenzialität einer elterlichen Aneignung des stillgeborenen Fötus ausgerichtet ist. In einem Vorgespräch z. B. versucht die Hebamme die Schwangere mit dem Argument von einem Kaiserschnitt abzubringen, dass ihr „bewusstes Miterleben der Geburt für den Trauerprozess wichtig sei und adressiert sie so als Mutter, die um ihr verstorbenes Kind trauert" (Völkle 2021, S. 121). Die Frau entbindet vaginal, ${ }^{10}$ das Paar entscheidet sich dagegen, das in der 24. SSW Stillgeborene nach der Geburt anzuschauen. In einem Hinterzimmer richtet die Hebamme es dennoch her, um eine spätere Familialisierung zu gewährleisten. Sie erstellt Hand- und Fußabdrücke, kleidet, inszeniert und fotografiert das Stillgeborene. Die Aufbewahrung der Fotos in der Akte ermöglicht auch Jahre später eine ,familiale Aneignung“ und hält „die Erwartung aufrecht, dass diese Aneignung irgendwann geschehen soll“ (ebd., S. 123, Herv. im Orig.). Hebammen wirken also ,aktiv darauf ein, dass das verstorbene Wesen eben nicht als ,Abgang ', ,regelwidrige Geburt“ oder als Fehl- oder Totgeburt angesehen wird - sondern stets als verstorbenes Kind“" (Wettmann 2021, S. 289).

Solche Anerkennungsakte sind für Babys höherer Schwangerschaftswochen etabliert. Die Frage, welche und ,wie viel“ Personalisierung für die Betroffenen angemessen ist, stellt sich dennoch in jeder Situation aufs Neue und noch einmal besonders bei Verlusten zu früheren Schwangerschaftszeitpunkten.

Im Hinblick auf die Deutung einer Geburt ambivalenter ist der Fall der Interviewten Kathleen, bei der in der 14. SSW eine Fehlgeburt diagnostiziert wird. Im Krankenhaus wartet sie über acht Stunden angeblich auf ein freies Zeitfenster für die Operation zur Ausschabung und erhält, ohne es zu wissen, wehenfördernde Mittel.

10 Viele Betroffene haben nach der Diagnose „Intrauteriner Fruchttod“ den Impuls, die tote Leibesfrucht durch einen Kaiserschnitt „loszuwerden“. Allerdings muss die Frucht etwa ab der 12. SSW in der Regel vaginal geboren werden, anschließend erfolgt gegebenenfalls eine Absaugung von Rückständen. 
Als sie im Bett liegend merkt: „das Kind ist rausgekommen“, traut sie sich nicht, unter die Decke zu schauen. Von der Situation überrascht und überfordert, ruft sie die Ärztin und bittet sie, das Fehlgeborene zu entfernen. Erst im Nachhinein wird Kathleen aufgeklärt, dass von vornherein geplant gewesen sei, dass sie auf natürlichem Wege gebäre. Kathleen war entsprechend unvorbereitet: „Bis zum Schluss wusste ich ja nicht, das ist das Label Geburt. Das habe ich ja erst zum Schluss gecheckt, okay, das war jetzt die Geburt.“ Ihr wurde, wie sie kritisiert, durch ,diese Verarschung“ die Möglichkeit genommen, den Prozess selbstbestimmt zu gestalten und ihrem Partner rechtzeitig Bescheid zu sagen.

Nach der Geburt hingegen erfolgen eine umfassende Aufklärung und Angebote der Personalisierung und Verabschiedung durch eine Krankenschwester (!), die sich direkt nach der unerwarteten Entbindung um das Fehlgeborene kümmert und Kathleen fragt, ob sie ,es“ sehen möchte. Diese lehnt zunächst ab, aber die Krankenschwester argumentiert behutsam dagegen. Sie erläutert „Vor- und Nachteile ..., wenn ich sehe oder nicht sehe“, berichtet aus eigener Erfahrung (,gut für den Verarbeitungsprozess“) und nimmt ihr die Scheu: „Sie meint, das sieht halt ganz süß aus und es sieht aus wie ein ganz normales Kind“. Für die Ermutigung ist Kathleen dankbar und betont, die Krankenschwester sei „wirklich richtig toll“" gewesen. Nachdem Kathleen sich rückversichert, dass die Krankenschwester den Fötus sofort entfernt, falls sie es wünscht, werfen sie und ihr Freund ein Blick auf das Fehlgeborene. Den ambivalenten Anblick beschreibt sie so:

Und das war/hat/es war halt/sah halt aus wie ein Kind. Also wie halt ein Babykind, ne. Wie wie ein Minikind.

Sie findet „ganz gut“, „es“ angeschaut zu haben, nimmt aber gegenüber dem Fötus keine elterliche Rolle ein. Vielmehr dekonstruiert die Beschreibung eine kindliche Personalität: Es sieht aus „wie ein Minikind“, ist aber keines. Andere Personalisierungen, wie Fotografien, nimmt sie nicht in Anspruch. Nachdem ihr die Bestattungsoptionen aufgezeigt worden sind, lehnt sie eine individuelle Beisetzung ab. Sie verzichtet auch auf den Besuch der Beerdigung:

Diese nette Hebamme [...] hat auch dafür plädiert und meinte, es sei gut für's Verabschieden und [...] jeder hat das Recht auf Trauer, ob das Kind jetzt eine Woche alt war oder neun Monate. Ich ne/ich dürfte das wahrnehmen. (1) Und das war aber so'n Gedanke, [...] so'n Todeszimmer, also so 'ne Trauerfeier mit ganz vielen weinenden Eltern, so hab' ich mir das vorgestellt, um mich herum nee, dachte ich mir irgendwie, das ist mir jetzt zu viel (2) [...], weil das sind halt auch Kinder, die ähm Totgeburten sind und so und später geboren werden. Die sind dann auch bei diesem, an dem Tag da. Und dachte ich mir irgendwie: ach nee, irgendwie kann ich das nicht. Die sind dann/die haben irgendwie mehr Recht auf Trauer oder so. Das ist irgendwie/dann bin ich da irgendwie mit meinem/mit meinem 14-Wochen-Kind da. Das ist irgendwie nicht so richtig. So. Aber auch diese Todesszenario-Situation, das war mir, glaub ich, zu viel.

Kathleen nimmt eine ambivalente Position in Bezug auf ihr fehlgeborenes Kind ein. Einerseits macht sie eine „Verlusthierarchie“ auf (vgl. Lovell 1983, S. 755), indem sie „Kinder“ „Totgeburten“ und ihr „14-Wochen-Kind“ unterscheidet. Damit 
reproduziert sie die Trauernorm, nach der offizielle Abschiedsrituale trauernden Eltern vorbehalten sind, deren Kinder später gestorben sind, und betrachtet sich selbst nicht als Teil der Community. Andererseits impliziert sie eine elterliche Beziehung zu ihrem Kind (,ich da mit meinem 14-Wochen-Kind“), die vergleichbar ist mit der der anderen Anwesenden. Sprachlich formen Kinder und Eltern hier eine Symbiose (,Totgeburten ... haben mehr Recht auf Trauer"). Auch wenn die imaginierte „Todesszenario-Situation“ für sie nicht infrage kommt, eignet sie sich den Rat zu elterlichen Verlustgefühlen und einem Abschiedsritual teilweise an.

Kathleen und Rhea bezeichnen sich nicht als Eltern. Sie sprechen distanzierend von der Geburt und dem Kind, dem sie keinen Namen geben. Sie besuchen weder die durch die jeweilige Klinik organisierte Trauerfeier, zu der sie eingeladen werden, noch das Sammelgrab. Beide beziehen sich aber positiv auf die Aufklärung und die Angebote zur personalisierenden Abschiednahme, die auf die rechtlichen Änderungen seit 2013 (wie die gesetzliche Verankerung einer Hinweispflicht für behandelnde Einrichtungen) zurückgehen. Kathleen und Rhea, wie auch das Paar in der Arbeit von Völkle (2021), sind seitdem in der Position, über Angebote der Personalisierung zu entscheiden und diese gegebenenfalls abzulehnen. Ihnen wird eine Selbstbestimmung über das eigene Stillgeborene zuteil, die im klinischen Kontext ein historisches Novum darstellt.

\section{Fazit und Ausblick}

Mit Fehlgeburt und Stillgeburt gehen Leiderfahrungen einher, die kulturell bedingt und somit vermeidbar sind. Ausgehend von der Kritik an fehlender Sichtbarkeit des Verlusts, an empathischem Versagen und an Entrechtungen engagieren sich seit nunmehr 40 Jahren Initiativen für Veränderungen. Einige Aktivist*innen engagieren sich für Anerkennung über den Versuch, einen eindeutigen Status für Fehlgeborene zu etablieren. Ein Ausdruck des Wandels ist die Verwendung der zunächst bewegungsinternen Bezeichnungen „Sternenkinder“ und „Sterneneltern“ durch offizielle Behörden. Im Beitrag wurde der ehrenamtlich forcierte Anerkennungswandel darüber hinaus anhand rechtlicher, medialer und geburtshilflicher Veränderungen im Umgang mit Fehl- und Stillgeburt beschrieben. Anhand eines Fallvergleiches wurde aufgezeigt, dass Angebote der Geburtsbegleitung und Personalisierung bei frühen Fehlgeburten (noch) weniger etabliert sind als bei späten Fehlgeburten bzw. Stillgeburten.

Es wurde dabei auch sichtbar, dass das mit den Gesetzesänderungen verbundene Narrativ entrechteter Elternschaft nicht für alle Betroffene passend ist. Vielmehr lassen sich Umgangsweisen auf zwei Polen ausmachen: Auf der einen Seite gibt es kollektive Anstrengungen von „Sterneneltern“, die eine Fehl- und Stillgeburt als Geburt ihres Kindes erfahren haben und als solche auch offiziell hätten anerkannt wissen wollen. Diesem Narrativ entsprechen die Gesetzesänderungen, gemeinschaftliche Formen des Gedenkens im Internet und auf Friedhöfen, mediale Diskurse auf „Mutterblogs“ und die Hebammenpraxis einer potenziellen Personalisierung des Stillgeborenen. Dem stehen auf der anderen Seite private Umgangsweisen entgegen, die sich in den narrativen Einzelinterviews zeigten. Während jene über materielle 
Fixierungen - von Ultraschallbildern über Videodokumentation bis zur staatlichen Bescheinigung - die Geburt (und den Verlust) ihres verstorbenen Kindes sichtbar und sozial geltend machen, dekonstruieren andere, wie Rhea und Kathleen, eine Personalität des Stillgeborenen und grenzen sich von einer Gemeinschaft der „Sterneneltern“ ab. Die beobachtbare Tendenz in Richtung einer öffentlichen Anerkennung von „Sternenkindern“ scheint im Privaten also weniger eindeutig zu sein. Es besteht Forschungsbedarf hinsichtlich der genauen Beweg- und Hintergründe von Akteur*innen, die eine offizielle Anerkennung und Beurkundung ihres verstorbenen Kindes einfordern. Denkbar ist, dass der Anspruch besonders in Milieus mit traditionellen Geschlechterrollen und Familienformen artikuliert wird, in denen ungewollte Kinderlosigkeit eine gewichtige Störung im ,normative life-course“ (Earle 2014, S. 151) ist.

Der multidimensionale Anerkennungswandel, Fehl- und Stillgeborene zu personalisieren und Betroffene als Eltern zu behandeln, wirft darüber hinaus Fragen nach Konsequenzen einer ,"Vereindeutigung“ in Richtung fötaler Elternschaft auf (vgl. Böcker 2021). Die Schaffung einer rechtlichen Möglichkeit zur Bestattung und zur Beurkundung von Fehlgeborenen wurde auch als Steigerung individueller Selbstbestimmung rezipiert: Es werde eine Möglichkeit, keine Pflicht, für diejenigen geschaffen, die sich als Eltern identifizieren und ihr Stillgeborenes bestatten und namentlich beurkunden wollen. Auf einen zweiten Blick ist es komplizierter. Bestrebungen zur Anerkennung einer personalen Existenz von Stillgeborenen und Darstellungen einer ,auf den Fötus zentrierten Trauer“ (Millar 2020, S. 100) liegen rhetorisch nahe an den Anliegen einer Bewegung, deren Anhänger*innen ungeborenen Föten einen Status als Rechtsperson verleihen wollen, um das fötale „Recht auf Leben“"gegen Selbstbestimmungsrechte auszuspielen. Wie beide Bewegungen konkret zusammenhängen, und wie anschlussfähig der Diskurs um Anerkennung von „Sterneneltern“ für rechtskonservative Anliegen ist, sind empirisch offene Fragen.

Danksagung Dank gilt allen Gesprächspartner*innen, die ihre Erfahrungen von Fehl- und Stillgeburt mit mir geteilt haben. Lea Gscheidel verdanke ich inhaltliche Hinweise. Janna Vogl sowie den beiden Gutachter*innen danke ich für die wertvolle Kritik am Manuskript, Alexander Leistner einmal mehr für seinen Blick auf das große Ganze und die geteilte Freude am Feinschliff.

Funding Open Access funding enabled and organized by Projekt DEAL.

Open Access Dieser Artikel wird unter der Creative Commons Namensnennung 4.0 International Lizenz veröffentlicht, welche die Nutzung, Vervielfältigung, Bearbeitung, Verbreitung und Wiedergabe in jeglichem Medium und Format erlaubt, sofern Sie den/die ursprünglichen Autor(en) und die Quelle ordnungsgemäß nennen, einen Link zur Creative Commons Lizenz beifügen und angeben, ob Änderungen vorgenommen wurden.

Die in diesem Artikel enthaltenen Bilder und sonstiges Drittmaterial unterliegen ebenfalls der genannten Creative Commons Lizenz, sofern sich aus der Abbildungslegende nichts anderes ergibt. Sofern das betreffende Material nicht unter der genannten Creative Commons Lizenz steht und die betreffende Handlung nicht nach gesetzlichen Vorschriften erlaubt ist, ist für die oben aufgeführten Weiterverwendungen des Materials die Einwilligung des jeweiligen Rechteinhabers einzuholen.

Weitere Details zur Lizenz entnehmen Sie bitte der Lizenzinformation auf http://creativecommons.org/ licenses/by/4.0/deed.de. 


\section{Literatur}

BMFSFJ. 2018. Sternenkinder. Hintergrundmeldung. https://www.bmfsfj.de/bmfsfj/themen/familie/ chancen-und-teilhabe-fuer-familien/sternenkinder. Zugegriffen: 21. Juni 2021.

BMJV, und BfJ. 2021. Verordnung zur Ausführung des Personenstandsgesetzes (Personenstandsverordnung - PStV) § 31 Lebendgeburt, Totgeburt, Fehlgeburt. https://www.gesetze-im-internet.de/pstv/_ 31.html. Zugegriffen: 21. Juni 2021.

Böcker, Julia. 2017. Inwändig, unsichtbar, liminal. Ambivalenzen pränataler Verluste. In Zur Soziologie des Sterbens. Aktuelle theoretische und empirische Beiträge, Hrsg. Nina Jakoby, Michaela Thönnes, 135-156. Wiesbaden: Springer VS.

Böcker, Julia. 2021. Mutter, Vater, Sternenkind. Die Änderung des Personenstandsrechts für Fehlgeborene und ihre unvorhergesehenen Folgen. In Facetten des Antifeminismus. Angriffe und Eingriffe in Wissenschaft und Gesellschaft, Hrsg. Johanna Sigl, Katharina Kapitza, und Karin Fischer, 117-133. Hamburg: MartaPress.

Böcker, Julia. 2022. Fehlgeburt und Stillgeburt. Eine Kultursoziologie der Verlusterfahrung. Weinheim, Basel: Juventa. im Erscheinen.

Brierley-Jones, Lyn, Rosalind Crawley, Samantha Lomax, und Susan Ayers. 2015. Stillbirth and stigma: the spoiling and repair of multiple social identities. OMEGA-Journal of Death and Dying 70(2):143-168. https://doi.org/10.2190/OM.70.2.a.

Bundeskanzleramt. 2019. Stille Geburt oder Tod des neugeborenen Kindes. Für Mütter und Väter, die von diesem Verlust betroffen sind. (Broschüre) Wien. https://www.bundeskanzleramt.gv.at/dam/jcr: 097caa2b-d17b-4969-b7d7-5681aac915b8/stille_geburt_broschuere.pdf. Zugegriffen: 17. Jan. 2022.

Butler, Judith. 2010. Raster des Krieges. Warum wir nicht jedes Leid beklagen. Frankfurt am Main, New York: Campus.

Carlson, Rose, Catherine Lammert, und Joann M. O’Leary. 2012. The evolution of group and online support for families who have experienced perinatal or neonatal loss. Illness, Crisis \& Loss 20(3):275-293. https://doi.org/10.2190/IL.20.3.e.

Cecil, Rosanne. 1994. Miscarriage: women's views of care. Journal of Reproductive and Infant Psychology 12(1):21-29. https://doi.org/10.1080/02646839408408864.

Côté-Arsenault, Denise, und Karen Kavanaugh. 2018. Editorial. Illness, Crisis \& Loss 26(1):3-4. https:// doi.org/10.1177/1054137317740797.

Dein-Sternenkind Stiftung. 2021. Webauftritt der Stiftung. https://www.dein-sternenkind.eu/. Zugegriffen: 27. Sept. 2021.

DEVA. 2021. Webauftritt der Initiative „Das Ende vom Anfang“ von Julia Stelzner-Konrad. https://www. dasendevomanfang.de/ueber. Zugegriffen: 27. Sept. 2021.

Doka, Kenneth J. (Hrsg.). 2002. Disenfranchised grief: new directions, challenges, and strategies for practice. Champaign: Research Press.

Duden, Barbara. 2002. Zwischen ,wahrem Wissen“ und Prophetie. Konzeptionen des Ungeborenen. In Geschichte des Ungeborenen. Zur Erfahrungs- und Wissenschaftsgeschichte der Schwangerschaft, 17.-20. Jahrhundert, Hrsg. Barbara Duden, Jürgen Schlumbohm, und Patrice Veit, 11-48. Göttingen: Vandenhoeck \& Ruprecht.

Earle, Sarah. 2014. A life-course approach to human reproduction. Human Fertility 17(3):151-153. https:// doi.org/10.3109/14647273.2014.940230.

Erhard, Franz, und Kornelia Sammet. 2018. Methodologische Grundlagen und praktische Verfahren der Sequenzanalyse. Eine didaktische Einführung. In Sequenzanalyse praktisch, Hrsg. Franz Erhard, Kornelia Sammet, 15-72. Weinheim, Basel: Juventa.

Faust, Julia. 2021. Auch tote Kinder werden groß. In Kinderkriegen. Reproduktion reloaded, Hrsg. Barbara Peveling, Nikola Richter, 114-127. Hamburg: Nautilus.

Franklin, Sarah. 1991. Fetal fascinations: new dimensions to the medical-scientific construction of fetal personhood. In Off-centre: feminism and cultural studies, Hrsg. Sarah Franklin, Celia Lury, und Jackie Stacey, 190-205. London: HarperCollins.

Frost, Julia, Harriet Bradley, Ruth Levitas, Lindsay Smith, und Jo Garcia. 2007. The loss of possibility: scientisation of death and the special case of early miscarriage. Sociology of Health \& Illness 29(7):1003-1022. https://doi.org/10.1111/j.1467-9566.2007.01019.x.

Graumann, Sigrid. 2011. Anerkennung und Sorgebeziehungen. In Akteur - Individuum - Subjekt: Fragen $z u$ „Personalität“ und „Sozialität“, Hrsg. Nico Lüdtke, Hironori Matsuzaki, 385-400. Wiesbaden: Springer VS. 
Gscheidel, Lea, und Clarissa Schwarz. 2021. Sternenkinder bestatten. Deutsche Hebammen Zeitschrift 73(1):36-39.

Han, Sallie. 2019. Pregnant with ideas. Concepts of the fetus in the twenty-first-century United States. In The anthropology of the fetus. Biology, culture, and society, Hrsg. Sallie Han, Tracy K. Betsinger, und Amy B. Scott, 59-80. New York, Oxford: Berghahn.

Hansen-Krewer, Corinna. 2021. Stille Geburten sind auch Geburten und Sterneneltern sind auch Eltern. Norderstedt: Books on Demand.

Heimbach, Birgit. 2021. Neue Töne zur Stillen Geburt. Deutsche Hebammen Zeitschrift 73(1):1.

Heimerl, Birgit. 2013. Die Ultraschallsprechstunde. Eine Ethnographie pränataldiagnostischer Situationen. Bielefeld: transcript.

Hirschauer, Stefan, Birgit Heimerl, Anika Hoffmann, und Peter Hofmann. 2014. Soziologie der Schwangerschaft: Explorationen Pränataler Sozialität. Stuttgart: Lucius \& Lucius.

Initiative Regenbogen e. V.. 2021. Wir sind. http://initiative-regenbogen.de/ueber-unsere-initiative.html. Zugegriffen: 24. Juni 2021.

Knöfel, Ulrike. 2020. Neue Bilder der Trauer. https://www.spiegel.de/kultur/chrissy-teigen-und-johnlegend-wie-das-paar-mit-dem-tod-ihres-sohns-umgeht-neue-bilder-der-trauer-a-81b3da30-0025432d-b8d9-dae207efd58f. Zugegriffen: 24. Juni 2021.

Köhne, Julia B. 2018. Absentes vergegenwärtigen: Schwangerschaftsabbruch und Fötalimagologie in westlichen Filmkulturen seit den 1960er-Jahren. In Sexualität und Widerstand: Internationale Filmkulturen, Hrsg. Aylin Basaran, Julia B. Köhne, Klaudija Sabo, und Christina Wieder. Wien, Berlin: Mandelbaum.

Kuberska, Karolina, Danielle Fuller, Jeannette Littlemore, Sheelagh McGuinness, und Sarah Turner. 2020. Death before birth: liminal bodies and legal frameworks. In A jurisprudence of the body, Hrsg. Chris Dietz, Mitchell Travis, und Michael Thomson, 149-178. Cham: Palgrave Macmillan. https://doi.org/ 10.1007/978-3-030-42200-4_7.

Lang, Ariella, Andrea R. Fleiszer, Fabie Duhamel, Wendy Sword, Kathleen R. Gilbert, und Serena CorsiniMunt. 2011. Perinatal loss and parental grief: the challenge of ambiguity and disenfranchised grief. OMEGA - Journal of Death and Dying 63(2):183-196. https://doi.org/10.2190/OM.63.2.e.

Layne, Linda L. 2000. 'He Was a Real Baby with Baby Things'. A material culture analysis of personhood, parenthood and pregnancy loss. Journal of Material Culture 5(3):321-345. https://doi.org/10.1177/ 135918350000500304.

Layne, Linda L. 2003. Motherhood lost: a feminist account of pregnancy loss in America. New York: Routledge.

Limbo, Rana. 2012. Caring for families experiencing stillbirth: a unified position statement on contact with the baby. Illness, Crisis \& Loss 20(3):295-298. https://doi.org/10.2190/IL.20.3.f.

Lovell, Alice. 1983. Some questions of identity. Late miscarriage, stillbirth and perinatal loss. Social Science \& Medicine 17(11):755-761. https://doi.org/10.1016/0277-9536(83)90264-2.

Lutz, Gottfried, und Barbara Künzer-Riebel (Hrsg.). 1989. Nur ein Hauch von Leben. Eltern berichten vom Tod ihres Babys und von der Zeit ihrer Trauer. Lahr: Ernst Kaufmann.

Martin, Barbara, und Marion Martin. 2014. Fest im Herzen lebt ihr weiter: Wie wir drei Kinder verloren und den Kampf um ihre Würde gewannen. Ein Ratgeber für Eltern von Sternenkindern. Asslar: Adeo.

Millar, Erica. 2020. Abtreibung. Eine Bestandsaufnahme. Bonn: Bundeszentrale für politische Bildung.

Oevermann, Ulrich, Tilman Allert, Elisabeth Konau, und Jürgen Krambeck. 1979. Die Methodologie einer „objektiven Hermeneutik“ und ihre allgemeine forschungslogische Bedeutung in den Sozialwissenschaften. In Interpretative Verfahren in den Sozial- und Textwissenschaften, Hrsg. Hans-Georg Soeffner, 352-433. Stuttgart: Metzler.

Peelen, Janneke. 2009. Reversing the past: monuments for stillborn children. Mortality 14(2):173-186. https://doi.org/10.1080/13576270902808043.

Petchesky, Rosalind Pollack. 1987. Fetal images: the power of visual culture in the politics of reproduction. Feminist Studies 13(2):263-292. https://doi.org/10.2307/3177802.

Peter, Claudia. 2013. Ungewissheiten in der ,Ankunft' eines frühgeborenen Kindes: Wahrnehmungen der Beteiligten. In Wissen an der Grenze. Zum Umgang mit Ungewissheit und Unsicherheit in der modernen Medizin, Hrsg. Claudia Peter, Dorett Funcke, 459-507. Frankfurt am Main, New York: Campus.

Peter, Claudia, und Dominik Feith. 2021. Familie und komplexe Schwangerschaften. In Handbuch Familie, Hrsg. Jutta Ecarius, Anja Schierbaum. Wiesbaden: Springer VS. https://doi.org/10.1007/978-3-65819861-9_43-3.

Przyborski, Aglaja, und Monika Wohlrab-Sahr. 2008. Qualitative Sozialforschung. Ein Arbeitsbuch. München: Oldenbourg. 
Rafanell, Irene, und Maja Sawicka. 2020. Emotions in digital interactions. Ethnopsychologies of angels' mothers in online bereavement communities. Cham: Palgrave Pivot.

Ramirez, Faustine D., Jori F. Bogetz, Megan Kufeld, und Lynn M. Yee. 2019. Professional bereavement photography in the setting of perinatal loss: a qualitative analysis. Global Pediatric Health https:// doi.org/10.1177/2333794X19854941.

Rando, Therese A. (Hrsg.). 1986. Parental loss of a child. Champaign: Research Press.

Sänger, Eva. 2020. Elternwerden zwischen „Babyfernsehen“ und medizinischer Überwachung. Eine Ethnografie pränataler Ultraschalluntersuchungen. Bielefeld: transcript.

Sani, Livia, Anne-Charlotte Laurenti Dimanche, und Marie-Frédérique Bacqué. 2021. Angels in the clouds: stillbirth and virtual cemeteries on 50 YouTube videos. OMEGA-Journal of Death and Dying 82(4):587-608. https://doi.org/10.1177/0030222818824732.

Schweizerische Eidgenossenschaft. 2017. Verbesserung der zivilstandsamtlichen Behandlung Fehlgeborener. Bericht des Bundesrates zum Postulat 14.4183 Streiff-Feller. https:/www.parlament.ch/centers/ eparl/curia/2014/20144183/Bericht\%20BR\%20D.pdf. Zugegriffen: 23. Juni 2021.

Strauss, Anselm. 1998. Grundlagen qualitativer Sozialforschung. München: Wilhelm Fink.

Strübing, Jörg. 2014. Grounded Theory und Theoretical Sampling. In Handbuch Methoden der empirischen Sozialforschung, Hrsg. Nina Baur, Jörg Blasius, 457-472. Wiesbaden: Springer VS.

Thompson, Neil, und Kenneth J. Doka. 2017. Disenfranchised grief. In Handbook of the sociology of death, grief, and bereavement. A guide to theory and practice, Hrsg. Neil Thompson, Gerry R. Cox, 177-190. New York, London: Routledge.

Traue, Boris, und Anja Schünzel. 2014. Visueller Aktivismus und affektive Öffentlichkeiten: Die Inszenierung von Körperwissen in „Pro Ana“- und „Fat Acceptance“-Blogs. Österreichische Zeitschrift für Soziologie 39:121-142. https://doi.org/10.1007/s11614-014-0134-6.

Verein Pusteblume. 2014. Die Petition ist am Weg! https://www.verein-pusteblume.at/petition-ist-amweg/. Zugegriffen: 3. Okt. 2021.

Villa, Paula-Irene, Stephan Moebius, und Barbara Thiessen (Hrsg.). 2011. Soziologie der Geburt: Diskurse, Praktiken und Perspektiven. Frankfurt am Main: Campus.

Völkle, Laura. 2021. Die Existenzweisen eines Fötus. Eine Einzelfallanalyse zu Prozessen der De/ Personalisierung und De/Humanisierung bei Totgeborenen. Zeitschrift für Soziologie 50(2):114-130. https://doi.org/10.1515/zfsoz-2021-0009.

Völkle, Laura, und Eva Muthmann. 2019. „Natürliche“ Kompetenzen in der Krise - Konstruktionen von Unfähigkeit beim Gebären und Stillen. Österreichische Zeitschrift für Soziologie 44:333-349. https:// doi.org/10.1007/s11614-019-00363-2.

Völkle, Laura, und Nico Wettmann. 2021. The process of pregnancy: paradoxical temporalities of prenatal entities. Human Studies-A Journal for Philosophy and the Social https://doi.org/10.1007/s10746021-09588-1.

Von der Hude, Kerstin, und Marion Glückselig. 2014. Familienzentrierte Trauerbegleitung. In Palliativversorgung und Trauerbegleitung in der Neonatologie, Hrsg. Lars Garten, Kerstin von der Hude, 113-144. Berlin, Heidelberg: Springer.

Warland, Jane, und Deborah L. Davis. 2011. Caring for families experiencing stillbirth. A unified position statement on contact with the baby. An international collaboration. http://missfoundation.org/ resources/news/StillbirthContactwBaby_position_statement.pdf. Zugegriffen: 23. Juni 2021.

Weilert, A. Katarina. 2017. Der rechtliche Rahmen für den Umgang mit Fehl- und Totgeburten. Rechtsmedizin 27(4):286-294. https://doi.org/10.1007/s00194-017-0172-z.

Werner-Lin, Allison, und Teresa Moro. 2004. Unacknowledged and stigmatized losses. In Living beyond loss. Death in the family, Hrsg. Froma Walsh, Monica McGoldrick, 247-271. New York: Norton.

Wettmann, Nico. 2021. „Geburt und Tod liegen ja sehr dicht beieinander“. Eine empirische Analyse zum pränatalen Tod im Denkstil von Hebammen. In Wissenssoziologie des Todes, Hrsg. Thorsten Benkel, Matthias Meitzler, 268-291. Weinheim, Basel: Juventa.

Wheeler, Inese. 1999. The role of linking objects in parental bereavement. OMEGA_Journal of Death and Dying 38(4):289-296. https://doi.org/10.2190/E2X1-TM7G-GDCQ-ULPW. 
Julia Böcker ist wissenschaftliche Mitarbeiterin am Institut für Soziologie und Kulturorganisation an der Leuphana Universität Lüneburg. Ihre Forschungsschwerpunkte sind Kultur- und Wissenssoziologie, Körper, Emotionen und Gesellschaft sowie qualitative Methodologie. Wichtige Veröffentlichungen: Fehlgeburt und Stillgeburt. Eine Kultursoziologie der Verlusterfahrung. Weinheim: Juventa (im Erscheinen). Alexander Leistner und Julia Böcker. 2021. „Im Osten geht die Sonne auf“ - Nostalgie als soziologische Erklärung der Gegenwart von Vergangenheit in Ostdeutschland? Zeithistorische Forschungen 1:133-139. Julia Böcker und Lena Dreier et al. (Hrsg.). 2018. Zum Verhältnis von Empirie und kultursoziologischer Theoriebildung. Stand und Perspektiven. Weinheim: Juventa. 